\title{
Direct to Consumer Pharmaceutical Advertising - Helpful or Harmful?
}

\author{
Sharifah Saffinas Syed Soffian ${ }^{1}$, Siti Maisara Amir ${ }^{1}$ and Faiz Daud ${ }^{1 *}$ \\ ${ }^{1}$ Faculty of Community Medicine, UKM Medical Center, Jalan Yaacob Latif, 56000 Bandar Tun Razak, Cheras, Kuala
}

Lumpur

\begin{abstract}
While direct-to-consumer advertising (DTCA) has been legalized in the United States and New Zealand, the prescribed drug marketing has long reached other places in the world including the Asian regions. Behind the successful profit-making pharmaceutical companies, concerns about public health impacts to the patient and physician counterpart have grown. Evidences illustrated that DTCA increases the volume of prescribing amount through patient demand and that the traditional manner of drug prescribing action has changed. However, the quality of health care trade in the relationship between physician and patient often overlooked and compromised. The systematic review analysed eight studies from five identified search engines using PRISMA guidelines. This review findings demonstrate DTCA implication in term of the health literacy, economic impact and relationship between physician and patient. Physician expressed substantial degree of dissatisfying behaviour when challenged with unnecessary need to prescribe drugs demand by patient. The prominent involvement of policymakers in regulating the guideline and controlling delivery of information in any advertisement related to drugs is direly important.
\end{abstract}

Keywords: Direct-to-consumer advertising; medical marketing; pharmaceutical; physicianpatient relationship; physician satisfaction

\section{INTRODUCTION}

Direct to Consumer Advertising (DTCA) is an attempt made by a corporation, mostly through mass media, to market its goods directly to patients or consumers (Lee, 2011). Globally, DTCA has becoming the current trend for many sectors including healthcare, even though United States (U.S) and New Zealand (N.Z) are currently the only countries which legalized DTCA (Lee, 2011; Schwartz \& Woloshin, 2019) The increasing demand for improved customer experiences, advanced technology and the acceptance of telemedicine among consumers are the selective reasons for the rise of DTCA (Jumpstart, 2019).

DTCA has been widely exploited in the healthcare products marketing strategy, ranging from medical devices and diagnostic equipment, health insurance, health services and pharmaceutical drugs (Friedman \& Gould, 2007). Numerous large pharmaceutical companies gained substantial income from DTCA despite the high capital spent to reach customers of different background. Literatures reviewed on the comparison of expenditure made for advertising activities between pharmaceutical companies and restaurant category (Macias, 2020). There was almost similar amount spent in both categories to accommodate for advertisement in various platform. The extensive drugs promotional efforts have been linked to medicalization issue among the viewers.

The expansion of DTCA-based marketing in medical field since the previous few decades alongside the rapid growth of technology was impressive (Cătoiu et al., 2013). The fascinating personalization element in the method used, urged patients to demand the advertised drugs from their physician. The advertisement influence patient to seek for medication by deviating the nonmedical problems to be defined as medical condition which may require pharmacological intervention (DeFrank et al., 2020). This in turn will pose more burden on the healthcare provider as the 
increasing demand for unnecessary drug prescription on top of existing patients in healthcare facilities.

Despite the ongoing debate on the implementation of DTCA by the pharmaceutical companies has towards patient as consumer, studies on the impacts toward the intermediary participant such as physician remains unclear. Traditionally, the physicians used to educate and prescribe medicines to the patients, but DTCA made it possible for patients to be informed directly through ad marketing. This would have certainly affected the communication to an extent. Hence, this review aimed to examine the impacts of DTCA and its detrimental effects on the relationship between physician and patient.

\section{MATERIALS AND METHOD}

Systematic search was performed for relevant articles between the year 2010 up to date from five search engines: PubMed, Sciencedirect, MedLine, Ovid and Scopus. We followed standard systematic review process as in the Preferred Reporting Items for Systematic Reviews and MetaAnalyses (PRISMA). PRISMA checklist guidelines was used for the workflow of publications search (refer Figure 1).

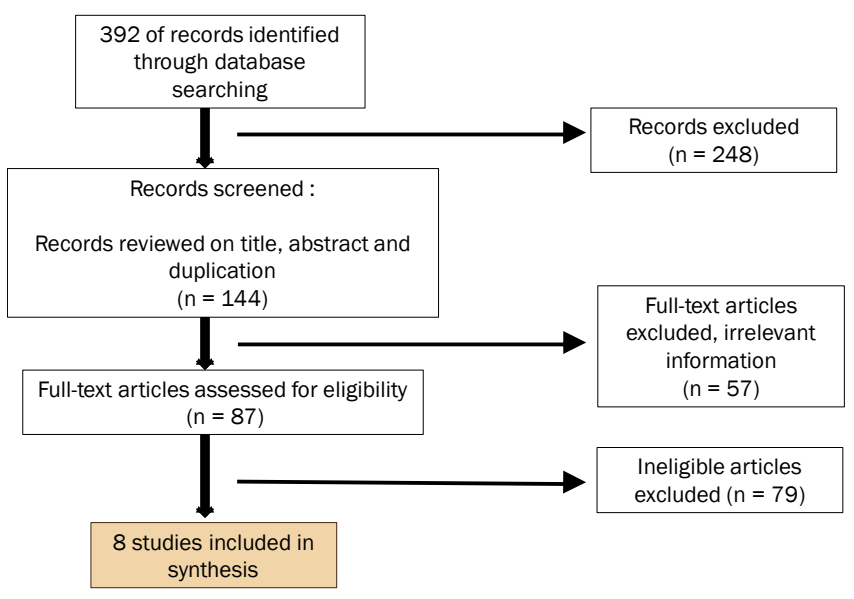

Figure 1. Flowchart of articles selection

The five major selected databases were accessed using the keywords "direct to consumer" AND "medical" OR "health" AND “marketing” OR "advertising" OR "promotion" OR "sell” AND "pharmaceutical" OR "medication" OR "remedy" OR "drug". For the articles identified from the database searches, we discarded articles that were duplicated in search engines before we screened them. We screened the title and abstracts of articles relevant to our research question. The selected articles were then assessed for availability in full text. Full text articles subsequently retrieved and evaluated based on inclusion and exclusion criteria. Inclusion criteria for included studies were: (a) articles published from the year 2010 to date, (b) articles studied on advertising product related to any type of medicine, drug, pharmaceutical or medication-like, (c) articles with topic of direct-to-consumer advertising (DTCA) including printed DTCA, online DTCA, eDTCA and (d) articles which focused on some aspect of DTCA, may it be the comparison effects, the impacts of DTCA, techniques used and demographics of various factors associated with DTCA. Studies were excluded if they are not published in English language, studies which did not underwent peer review, those of non-drugs related and of which focused solely on the cost analysis perspective. Total of five electronic database searches yielded eight articles for reviewing. [Table 1].

\section{RESULT AND DISCUSSION}

\section{A. Characteristics of Included Studies}

This systematic review includes articles from year 2010 to 2019 from various countries, particularly the United States and New Zealand where DTCA is legal in respective countries. Most studies conducted on DTCA used quantitative methods rather than qualitative. Out of the eight studies selected in this review, six of them used cross-sectional design while another two using qualitative method. Studies that targeted health care provider as their respondent were selected from physician and nurse prescriber with sample size $(n)$ ranges between 400 and 1,163. One study, Grenard et al. (2011) focuses on perceptions of the elderly towards effects of DTCA in the US. Two studies examined on the compliance of advertisement broadcasted through television channels and online platform, which has become more personalized nowadays. The identified advantages and disadvantages of DTCA in studies collated were further classified into three main perspectives.

\section{B. Health Literacy}

Marketing has the potential to substantially contribute to improving health outcomes. DTCA targets at individual level for medicinal drug promotion by providing information on 
the diseases related to usage of the drug. Five out of eight studies mentioned on the benefits gained by customers upon marketing through DTCA differ by the type of advertisement being utilized. Grenard et al. (2011) examined DTCA as an approach to enhance the existing knowledge of the population regarding general health matter. Two third of the physician in the United States agreed that patients seek for early medical attention on conditions they thought needful secondary to the information obtained through advertisement. Klara et al. (2018) described on the broadcast DTC advertisement for prescription drugs in the US with being able to empower and engage patients to participate in their own health care. Upon further examination of the ads content, the study highlighted on the health knowledge gained by patients. However, imbalanced and misleading information could cause confusion among the patient and that somewhat led to unnecessary prescriptions by their doctor. Majority of the advertisement misinformed the patients by over-emphasizing the treatment benefits to the risk and over-claimed drugs promotion over healthy lifestyle choices. Though DTCA believed to help improve the health literacy of general population, it does create unnecessary pressure on other social aspects including patient doctor relationship.

\section{Economic Impact}

In the US., spending on DTCA rose from US\$ 2.1 billion in 1997 to US\$ 9.6 billion in 2016, with pharmaceutical firms increasing from US\$ 1.3 billion to US\$ 9.6 billion spend to market their own medicines for different diseases (Schwartz \& Woloshin, 2019). Anna et al. (2019) revealed that among 1,163 nurse prescribers in the US, $80 \%$ believed that DTCA escalated the cost of prescription drugs. In marketing strategy by the pharmaceutical companies, when the demand is high, the opportunity to increase sales gets higher, hence the price would be worth increased. Grenard et al. (2011) measured the implication of increased financial cost in term extra consultation time needed to spend to correct the misconceptions perceived by patients in commercial DTCA. Indirectly it contributes to the raise of financial burden posed on healthcare system unnecessarily. More than half consumers in Korea thought that by having DTCA, the drug prices will be inflated and increase the revenue of large pharmaceutical companies (Suh, Lee, Kim, Chee, \& Kang, 2011). Despite DTCA has not been legalized in Korea, many of the consumers were aware of the marketing strategies accessible through the internet online advertisement and expressed their acceptance towards receiving such advertisement. Mintzes et al. (2012) conducted a review of the research evidence on the effects of DTCA towards drug sales and demand. They concluded that DTCA serves as an effective means of boosting sales for pharmaceutical companies. In one of the studies examined a 2-year campaign on toenail fungus via advertisement of terbinafine, had found that the prescriptions rate was increased by double. The benefits gained by the pharmaceutical companies were estimated at almost USD 11.5 million (Mintzes, 2012).

\section{Patient-Doctor Relationship}

DTCA changes the dynamic of the relationship between the doctor and patient. As such, many studies linked DTCA with disruption of patient-doctor relationship. A study conducted among physician in Jordan illustrated DTCA as posing a negative impact as well as causing harm to physician-patient relationship (Abo-Rumman \& Ahmad, 2012). Though DTCA is not legalised in Jordan but based on literature and exemplified by other supplemental food products, physician seek to maintain traditionally strong doctor-patient relationship. Anna et al. (2019) described DTCA has worsen nurse-patient relationship that they perceived patient request for certain drugs as inappropriate. Mikhael et al. (2013) demonstrated elements of emotions-driven theme widely used in DTC advertisement. Emotional appeals overshadowed the facts leading patients to seek for the drugs unnecessarily. Satisfaction among the healthcare provider is hampered by limited knowledge of the consumers in the sense that they have to prescribe drugs over emotional approach rather than evidence based treatment (Mikhael, 2013). Vast majority of the DTCA studied explained benefits in vague terms, often utilising personalised strategy whereby exaggerating selective information which mislead patients (Every-Palmer et al., 2014). This situation had put physician under pressure as to rectify information and that discriminate the trust in doctor-patient relationship. Some doctors were challenged when patients insisted on prescription of the drugs requested where indeed there is no 
indication. Nonetheless, physician contended to patient's demands often felt unsatisfied and expressed dilemma on ethical obligation to do so (Marimuthu, 2016).

There have been various types of DTCA, namely helpseeking ad, reminder ad and product claim ad. The helpseeking ad is categorized by the presence of information regarding the disease while omitting the information on drug. Meanwhile, the reminder ad consists of information on prescription medicine such as the name of a drug, price and strength but did not stated the indication or claim on efficacy of the drug effect. The other type is the product claim ad whereby it provides the indication, efficacy and safety profile of the prescription drug (Lee, 2011). While the authorities have outlined the criteria for pharmaceutical marketing advertisement explicitly, there has been inclining number of cases fined for breaching the act. Despite DTCA has not been legalized in majority of countries in the world, this strategy of drug promotional is rampant and most commonly used by large pharmaceutical drug companies. Many critics have addressed on the under regulation of the guidelines that urged for strengthening of rule and regulation posed on the marketers (Marimuthu, 2016).

The results obtained in this systematic review indicates the drawbacks outweighed the benefits of DTCA particularly on the healthcare provider and patient counterpart. Advertisement on prescribed drugs can influence patient's trust with their physician's clinical decisions (Filipova, 2019). Information delivered via the ads may lead patients to make their own conclusion based on limited knowledge and understanding. In turn, patients tried to argue with the physicians on choice of prescribed medications whereby demanding on the advertised drugs unnecessarily. Physician on the other hand, may find themselves challenged with increased burden of work and frustration on patient inquiries regarding their clinical judgement and authority over information gained by patients through DTCA and not evidence based. The mishaps had changed the physicianpatient relationship into a more emotional appeals approach rather than evidence-based medicine. Conflict commonly expressed by physician is on the ethical consideration of doing more harm against subscribe to patient's demands as the consumer, hampers the physician-patient relationship further. Mikhael et al. (2013) revealed high unsatisfactory among the physician towards the changing trend of relationship between doctor and patient into doctor and customer.

Out of spending on medical marketing of drugs, disease awareness programs, health services, and laboratory testing in U.S., DTCA had the most rapid growth of spending from 1997 through 2016 (Schwartz \& Woloshin, 2019). DTCA indirectly raises the cost of drugs and healthcare, which then affects end-users who need to spend the extra money (Limbu \& Mukherjee, 2016). As patient demand for advertised medicines, they tend to shift away from cheaper alternatives, such as generic medications which has same efficacy and more affordable. The raise of financial burden due to DTCA pose an economic impact to the healthcare system specifically.

DTCA pushes patients to prompt discussion with physician regarding medication and diseases that may go untreated (Parekh \& Shrank, 2018). It fosters good habit among the patients so that they could seek early treatment and improved health outcome. Patients receiving the DTCA-related medication showed 43 per cent higher satisfaction compared to those receiving other medicines (Preechavuthinant et al., 2017). However, many physicians faced difficulties and timeconsuming in the need to convince patients that a requested drug may not be necessary in certain conditions (AboRumman \& Ahmad, 2012). With the current heavy workloads in the government health care clinics, physician need to limit consultation time over other patients in order to educate and correct information. Besides that, this interrupts with physician-patient communication in a way that it leaves little time for health care provider to address some important issues regarding the patient condition. Discussion about advertised drugs can be prolonged and that diverted the purpose of routine follow up examinations (Lee, 2011). Doctors also resented frustration over being responsible to explain on advertised products rather than to focus on treatment.

The implementation of DTCA should involve multiple partnership participation from the policy maker, pharmaceutical companies, physicians and even the patients (Parekh \& Shrank, 2018). The regulatory authorities hold the obligation of quality control over information being delivered to the public via DTCA. The use of unbiased entity with 
pharmacological background as an independent body to review the compliance towards advertisement guideline is crucial. Furthermore, the lack in transparency of the content in prescribed drug advertisement is of concern. Pharmaceutical companies particularly shall improve on initiatives to increase the provision of accurate, accessible and reliable health information to the public as the ads may be misinterpreted by most of them. Physicians on the other hand need to take responsibility and create greater trust with patients in order to maximize the quality of future health care. Patients empowerment in terms of awareness and health seeking behaviour from the correct sources are important as they are the end user of the marketed products.

\section{CONCLUSION}

From this systematic analysis, the related studies concluded that disadvantages of DTCA exceeded the advantages. Even though DTCA helps to educate patient on illness and diseases, often the deceptive information prompt further discussion with physician. Therefore, may affects the motivation and satisfaction of physician when challenged. Against this backdrop, assumptions that DTCA enhances the relationship between physician and patient remains speculative.

Since DTCA is not legal in Malaysia, this should remain so as a health-protection measure towards the community. The policymakers are advised to enhance monitoring and apply strict penalty to pharmaceutical companies violating the rules and regulations of DTCA in order to avoid further complications.

\section{ACKNOWLEDGEMENT}

We would like to thank the Director General of Health Malaysia for his permission to publish the findings from this study. We also would like to thank the Dean of Faculty of Medicine, National University of Malaysia for his supports.

Friedman, M, \& Gould, J 2007, 'Physicians' attitudes toward direct-to-consumer prescription drug marketing', Journal of Medical Marketing, vol. 7, no. 1, pp. 33-44.

Jumpstart, F 2019, Market forces influencing the growth of the direct-to-consumer model in healthcare, viewed 20 May 2020, < < <tps://www.jsf.co/blog3/2019/3/13/marketforces-influencing-the-growth-of-the-direct-to-consumermodel-in-healthcare>.

Klara, K, Kim, J, \& Ross, JS 2018, 'Direct-to-Consumer broadcast advertisements for pharmaceuticals: off-label promotion and adherence to FDA guidelines', Journal of General Internal Medicine, vol. 1, pp. 1-8.

Lee, VC 2011, 'Direct-to-consumer pharmaceutical advertising therapeutic or toxic?', P and T, vol. 36 , no. 10 , pp. 669-684.

Limbu, Y, \& Mukherjee, A 2016, 'Direct-to-consumer advertising: a review and agenda for future research', Thriving in a New World Economy, Springer, pp. 177-180. Macias, W 2020, 'Why the "sea of same"? A qualitative look at DTCA within the larger context of healthcare marketing', Health Marketing Quarterly, pp. 1-21. 
Marimuthu, SB 2016, 'Should Direct to Consumer Advertisements (DTCA) of prescription drugs remain banned in Malaysia ?', Australian Journal of Asian Law, vol. 17 , no. 1, pp. 1-14.

Mikhael, EM 2013, 'Evaluation of the ethical issues for the direct to consumer advertisement in Arabic satellite channels', Global Research Analysis, vol. 2, no. 2277, pp. 141-143.

Mintzes, B 2012, 'Advertising of prescription-only medicines to the public: does evidence of benefit counterbalance harm?', Annual Review of Public Health, vol. 33, no. 1, pp. 259-277.

Paga, A, Frosch, DL, Grenard, JL, \& Uy, V 2011, 'Seniors' perceptions of prescription drug advertisements: a pilot study of the potential impact on informed decision making', Patient Education and Counseling, vol. 85, pp. 79-84.

Parekh, N \& Shrank, WH 2018, 'Dangers and opportunities of direct-to-consumer advertising', Journal of General Internal Medicine, pp. 586-587.

Preechavuthinant, S, \& William, KW 2017, 'The trends in DTCA and effects of DTCA by pharmaceutical firms in the United States', Business \& Health Administration Proceedings, pp. 286-295.

Schwartz, LM \& Woloshin, S 2019, 'Medical marketing in the United States, 1997-2016', JAMA - Journal of the American Medical Association, vol. 321, no. 1, pp. 80-96.

Suh, HS, Lee, D, Kim, SY, Chee, DH, \& Kang, HY 2011, 'Direct-to-consumer advertising (DTCA) for prescription drugs: consumers' attitudes and preferences concerning its regulation in South Korea', Health Policy, vol. 101, no. 3, pp. 260-268. 